\title{
Parâmetros de infiltrabilidade em solo urbano para modelo chuva-vazão
}

Parameters of infiltration rates in urban soil for rain-flow model

Parámetros de velocidad de infiltración en el suelo urbano para el modelo lluviaescorrentía

Marcos Antonio Barbosa da Silva Junior Mestre em Engenharia Civil, UPE, Brasil. marcos15barbosa@hotmail.com.br

Simone Rosa da Silva Professora Doutora, UPE, Brasil. simonerosa@poli.br

Willames de Albuquerque Soares Professor Doutor, UPE, Brasil. was@poli.br 


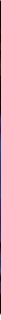

RESUMO

O presente trabalho consiste na obtenção dos parâmetros de infiltrabilidade em solo urbano, numa área da cidade do Recife (PE) com ocorrência de alagamentos, a partir da realização de ensaios com infiltrômetro a simples anel. A equação resultante da curva de infiltração foi ajustada ao modelo de infiltração de Horton, definindo-se os parâmetros de taxas máxima e mínima de infiltração, e constante de decaimento. Verificou-se que os parâmetros obtidos, quando utilizados em um modelo chuva-vazão, representaram bem as condições do balanço hídrico local, resultando num escoamento superficial (runnof) muito próximo dos padrões atuais de uso e ocupação do solo da cidade.

PALAVRAS-CHAVE: Infiltração. Solo urbano. Modelo chuva-vazão.

\section{ABSTRACT:}

The present work consists in obtaining the parameters of infiltration rates in urban soil, in an area in the city of Recife (PE) with occurrence of floods, from the performance of the tests with a simple ring infiltrometer. The resulting equation of the infiltration curve was adjusted to the Horton infiltration model, defining the parameters of maximum and minimum rates of infiltration, and decay constant. It was found that the parameters obtained, when used in a rain-flow model, represented well the conditions of the local water balance, resulting in a superficial flow (runoff) very similar to the city's current urbanistic patterns.

KEYWORDS: Infiltration. Urban soil. Rain-flow model.

\section{RESUMEN:}

Este trabajo consiste en conseguir los parámetros de velocidad de infiltración en el suelo urbano en una zona de Recife (PE) con la ocurrencia de inundaciones, a partir de los ensayos en simple anillo infiltrómetros. La curva de infiltración ecuación resultante se ajustó Horton modelo de infiltración, los parámetros que definen los tipos mínimos de la infiltración y la constante de desintegración y máximo. Se encontró que los parámetros obtenidos cuando se utiliza en un modelo lluvia- escorrentía y representado condiciones de equilibrio hídrico de la zona, lo que resulta en la escorrentía (runnof) muy cerca de los patrones actuales de uso y ocupación del suelo de la ciudad.

PALABRAS CLAVE: La infiltración. Suelo urbano. Modelo de lluvia- escorrentía. 


\section{INTRODUÇÃO}

A infiltração é definida como o processo inicial da entrada de água no solo, resultante da aplicação na superfície do solo, quer por chuvas ou por irrigação, onde a capilaridade é dominante nessa fase (SOARES, 2010).

A capacidade de infiltração varia para cada tipo de solo. Muitos métodos baseados na infiltração da água no solo foram desenvolvidos para determinar os principais parâmetros que se deseja conhecer num determinado solo estudado. Com a obtenção destes parâmetros, é possível conhecer, a partir de simulações hidrológicas em modelos chuva-vazão, a parcela de escoamento superficial gerado por um evento de precipitação intensa, numa determinada área de estudo.

Atualmente, há modelos hidrológicos específicos para as mais diversas áreas de estudo, tanto para áreas rurais, quanto para urbanas. Em áreas urbanas, o uso de modelos hidrológicos está associado principalmente ao planejamento da drenagem urbana, seja em maior ou menor escala espacial. Em maior escala, modelos hidrológicos podem ser empregados para a estimativa da geração do escoamento superficial em grandes bacias urbanas, sendo possível a simulação de distintos cenários de uso e ocupação do solo, de modo que as zonas de expansão urbana possam ser avaliadas. Em menor escala espacial, os modelos hidrológicos permitem, por exemplo, avaliar o impacto das BMP's (Best Management Practices), ou práticas de melhor manejo, no âmbito de lotes residenciais, como o uso de trincheiras de infiltração e reservatórios de amortecimento (BELING, 2013).

Quando o foco volta-se para as áreas urbanas, encontram-se diversos modelos capazes de reproduzir as peculiaridades encontradas nesses meios. Butler e Davies (2010) destacam os modelos SWMM (Storm Water Management Model), MIKE URBAN e HydroWORKS como exemplos para a aplicação em meios urbanos.

Dentre os modelos apresentados, o SWMM, desenvolvido em 1971 pela EPA-USA (Enviromental Protection Agency), é o pacote computacional mais utilizado para simulação da drenagem urbana, principalmente por tratar-se de um software livre, dispondo de seu código fonte aberto, colaborando assim para sua utilização em nível mundial nos vários centros de pesquisas. Desde o seu desenvolvimento, o algoritmo do SWMM vem sofrendo gradativas mudanças atualizando e/ou incluindo a simulação de distintos processos hidrológicos. Atualmente, a versão disponibilizada é a 5.0, contando com interface gráfica para a introdução dos dados de entrada.

No ambiente do sobredito modelo, os principais parâmetros de infiltração que influenciam diretamente na geração do escoamento superficial são: as taxas máxima e mínima de infiltração $(\mathrm{mm} / \mathrm{h})$ e a constante de decaimento $\left(\mathrm{h}^{-1}\right)$.

Assim sendo, o presente artigo define os citados parâmetros de infiltrabilidade numa parcela de solo urbano, fazendo uso do infiltrômetro a simples anel. Os ensaios foram realizados no bairro da Soledade, município do Recife, o qual foi escolhido por apresentar problemas de alagamentos durante os períodos de chuva. 


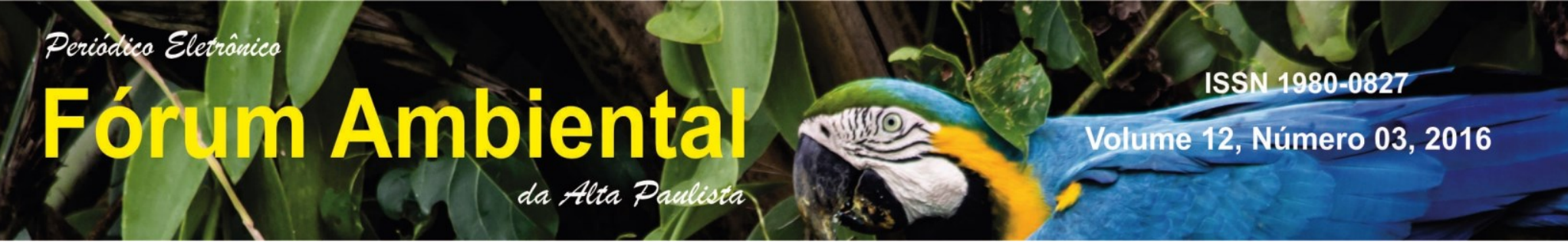

O sobredito ponto crítico de alagamento, localizado mais especificamente no cruzamento da Avenida João de Barros com a Rua Joaquim Felipe, foi objeto de estudo de Silva Junior (2015) que apresentou soluções mitigadoras para o problema, a partir dos resultados apontados pelas simulações no modelo SWMM. Assim, o desenvolvimento dos ensaios de infiltração e o tratamento dos resultados obtidos, os quais são discutidos neste artigo, subsidiaram parte dos dados de entrada que viabilizou a realização das modelagens no citado estudo.

\section{OBJETIVOS}

Diante das peculiaridades urbanísticas e geográficas apresentadas pela cidade, atualmente o Recife apresenta 159 pontos de alagamentos, catalogados pela Emlurb (Empresa de Manutenção e Limpeza Urbana), como os mais críticos. Dentro deste contexto, o presente trabalho levantou parâmetros de infiltrabilidade (taxas máximas e mínimas de infiltração, e constante de decaimento), a partir do infiltrômetro a simples anel, necessários para um estudo de alternativas mitigatórias para alagamentos, a partir de modelagens hidrológicas.

\section{METODOLOGIA / MÉTODO DE ANÁLISE}

O SWMM considera três modelos diferentes para a determinação da variável infiltração da chuva em zona não saturada do solo nas áreas permeáveis: Método de infiltração de Horton, Green-Ampt e Curva Número do SCS.

No presente artigo, o método de Horton foi empregado para a representação do processo de infiltração, considerando a facilidade de obtenção de seus parâmetros obtidos a partir dos ensaios de infiltração realizados na área em estudo.

Os ensaios foram concebidos numa parcela com solo sem vegetação em área próxima ao ponto de alagamento, a partir de cinco pontos dispostos na forma de um trapézio (Figura 1).

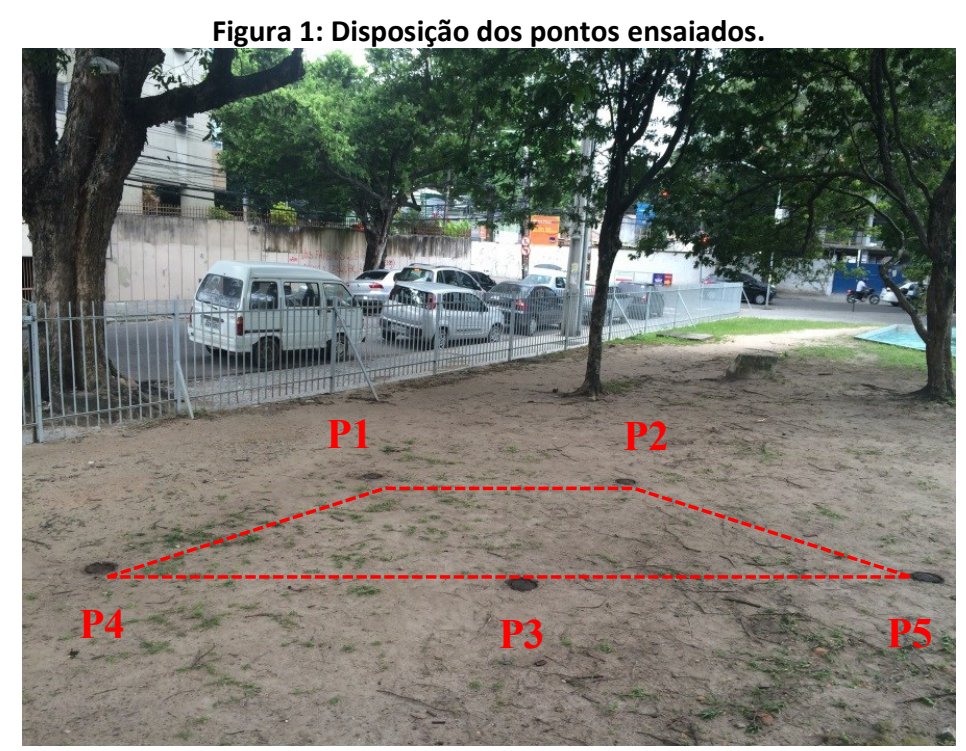


Figura 4: Curvas de infiltração nos cinco pontos ensaiados.

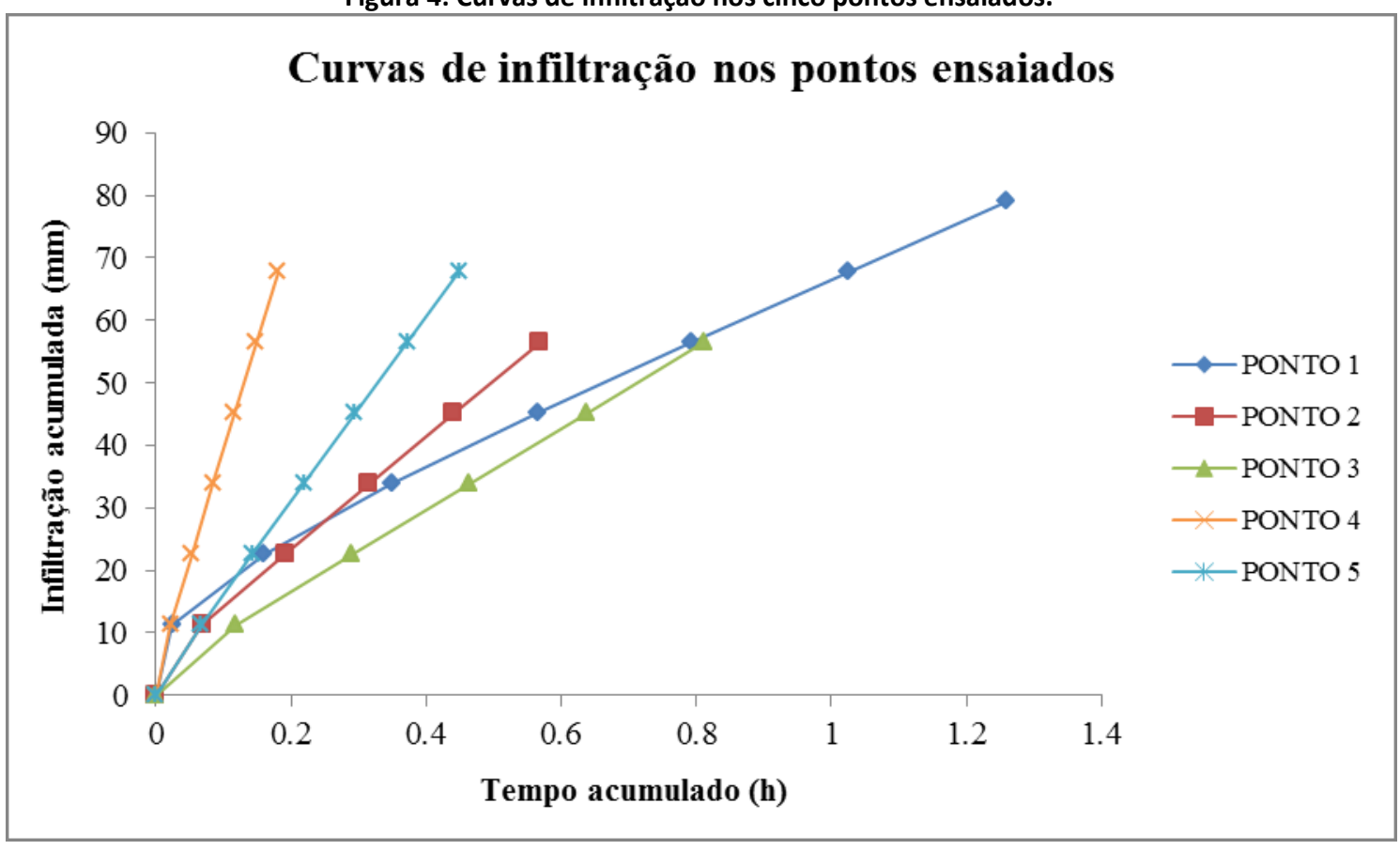

Figura 5: Curvas de capacidade de infiltração nos cinco pontos ensaiados e índices de correlação.

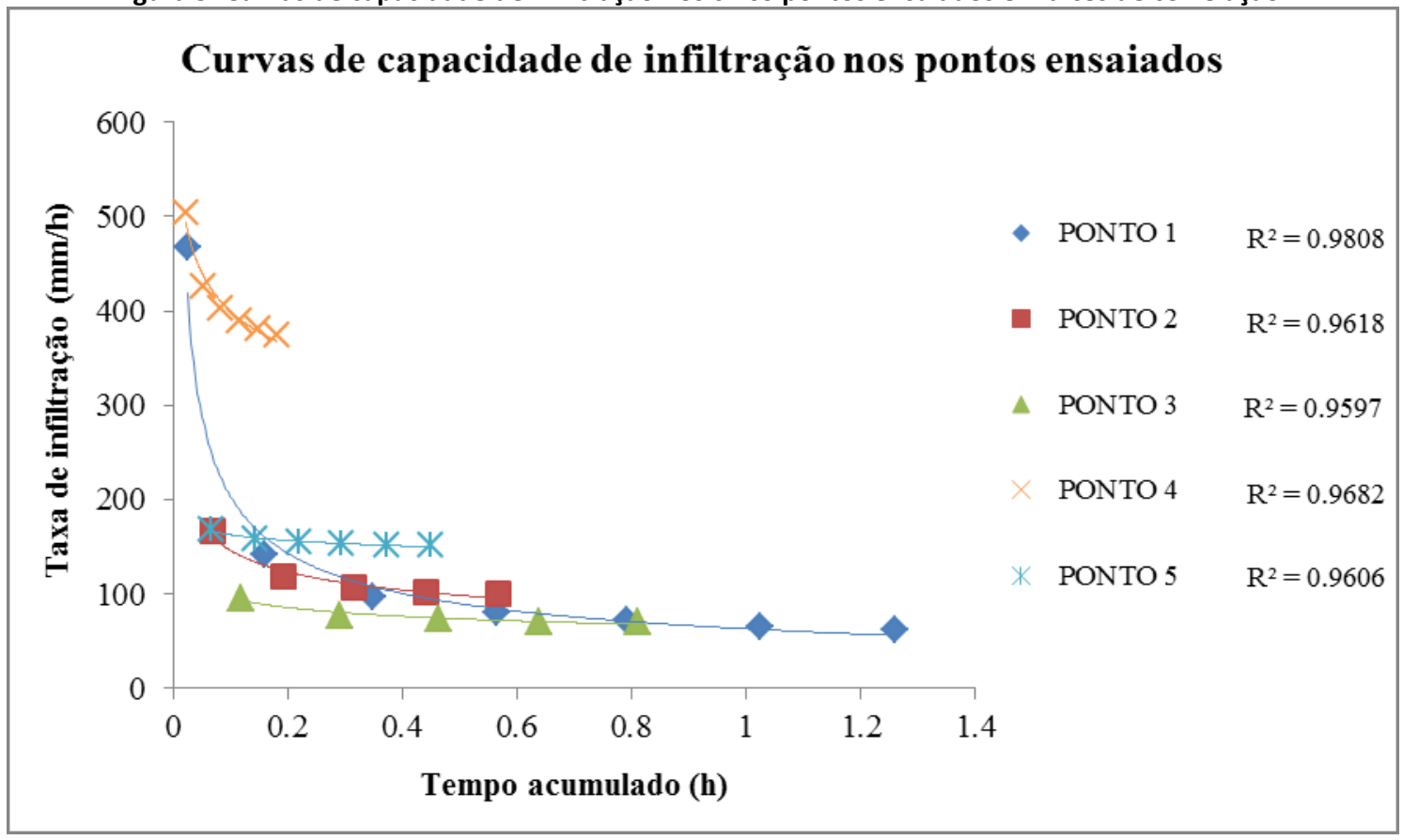

Apesar da proximidade entre os cinco pontos ensaiados, as curvas resultantes dos ensaios de infiltração apresentaram comportamentos heterogêneos, conforme observa-se no gráfico da Figura 4. Este fato pode está associado à compactação do solo nos pontos investigados e também às condições de umidade inicial do ensaio. 
Diante de tal constatação, resolveu-se utilizar a curva de capacidade de infiltração que apresentou melhor ajuste (Figura 5), com base no índice de correlação $\left(R^{2}\right)$. $O$ valor do coeficiente de determinação, descrito aqui, apenas diz o quanto a curva gerada pelo Excel conseguiu representar os pontos.

Assim, seguindo este critério, verificou-se que a curva de infiltração do Ponto 1 apresentou o índice de correlação mais próximo de $1\left(R^{2}=0.9808\right)$, com sua equação resultante mostrada na Figura 6.

Figura 6: Curva ajustada da taxa de infiltração no Ponto 1 e equação da curva.

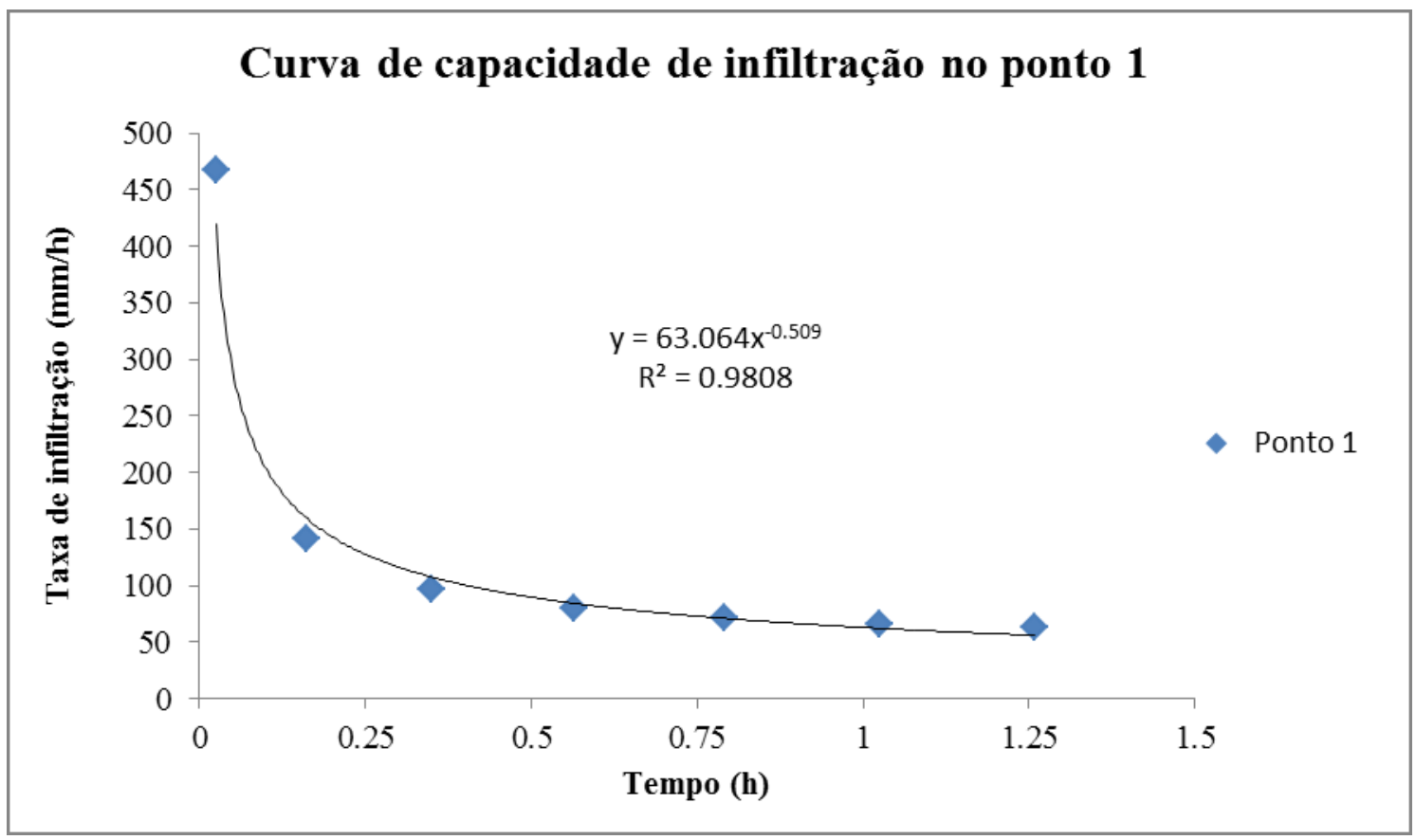

Com base na equação da curva de infiltração apresentada, sendo a mesma ajustada à equação de Horton (Equação 1), foi possível a obtenção dos parâmetros necessários para a alimentação do modelo: as taxas máxima e mínima de infiltração $(467,56 \mathrm{~mm} / \mathrm{h}$ e $62,79 \mathrm{~mm} / \mathrm{h}$, respectivamente), e a constante de decaimento (Equação 2).

$$
I t=I b+(l o-l b) \exp (-k \cdot t)
$$

Onde: It - taxa de infiltração no tempo $(\mathrm{mm} / \mathrm{h})$

$\mathrm{lb}$ - taxa de infiltração final $(\mathrm{mm} / \mathrm{h})$

lo - taxa de infiltração inicial $(\mathrm{mm} / \mathrm{h})$

$\mathrm{t}$ - tempo $(\mathrm{h})$

$k$ - constante de decaimento $\left(\mathrm{h}^{-1}\right)$

$$
k=(l o-l b) / F c
$$


Onde: $10-467,56 \mathrm{~mm} / \mathrm{h}$

$\mathrm{lb}-62,79 \mathrm{~mm} / \mathrm{h}$

Fc - é dado como a área sob a curva da taxa de infiltração, obtida a partir da integração da equação da curva ajustada (Figura 5), variando no intervalo de tempo de realização do ensaio (em horas), conforme mostrado a seguir.

$\begin{aligned} \mathrm{FC}=\int_{0,024}^{1,259} 63,06 \cdot \mathrm{X}^{-0,51} & =>128,694 \cdot \mathrm{X}^{0,49} \\ & =>128,694\left(\mathrm{t}_{\text {final }}{ }^{0,49}-\mathrm{t}_{\text {inicial }}{ }^{0,49}\right) \\ & =>128,694\left(1,259^{0,49}-0,024^{0,49}\right) \\ & \mathrm{FC}=123,34 \mathrm{~mm}^{2}\end{aligned}$

Com o Fc encontrado, fez-se uso da Equação 2 e obteve-se a constante de decaimento (k) de 3,282 .

De posse os parâmetros: taxas máxima e mínima de infiltração; e constante de decaimento, e com base na Equação 1, obteve-se a seguinte equação de Horton que estabelece a capacidade de infiltração na área estudada:

$$
\mathrm{I}_{\mathrm{t}}=62,79+404,77 \cdot \mathrm{e}^{-3,282 \mathrm{t}}
$$

Assim, uma vez obtidos os parâmetros de infiltração, que serviram de dados de entrada para as simulações hidráulica-hidrológicas realizados por Silva Junior (2015), a Figura 7 mostra a inserção destes parâmetros na plataforma do modelo SWMM.

Figura 7: Parâmetros de infiltração inseridos no modelo.

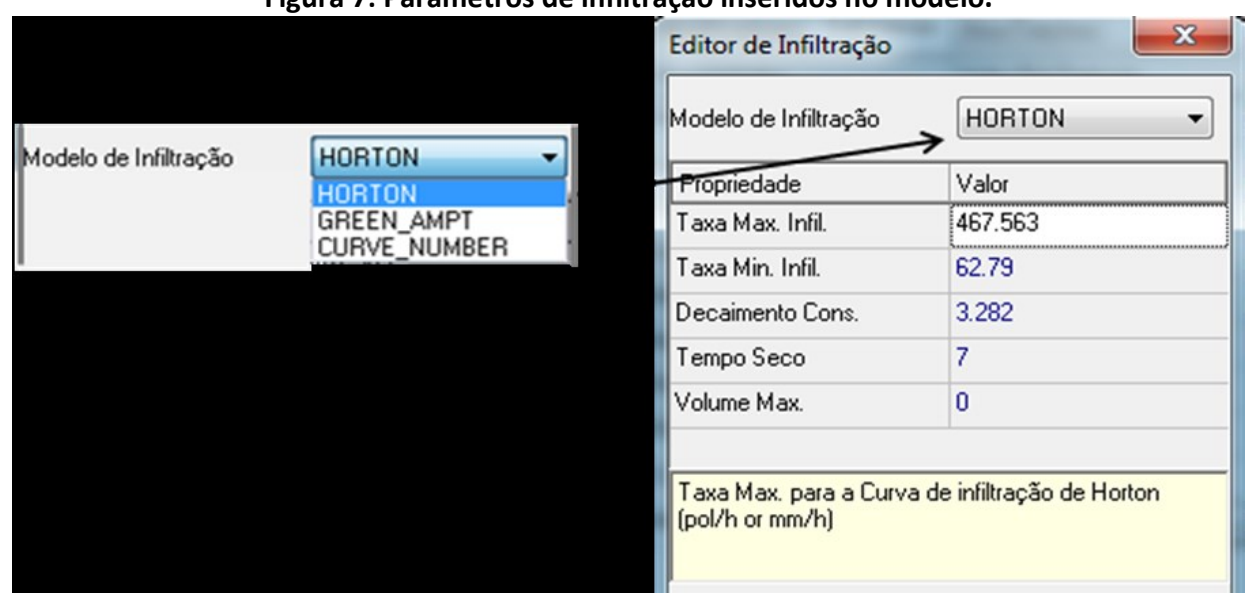


